\title{
Marked Underreporting of Pertussis Requiring Hospitalization in Infants as Estimated by Capture-Recapture Methodology, Germany, 2013-2015
}

\author{
Anika Schielke, PhD, *† + Anja Takla, MD, * Rüdiger von Kries, PhD, $\S$ Ole Wichmann, PhD, * \\ and Wiebke Hellenbrand, MD*
}

\begin{abstract}
Background: In Germany, pertussis became notifiable in eastern federal states in 2002 and nationwide in March 2013. Infants are at greatest risk for severe disease, with a high proportion requiring hospitalization. We implemented enhanced hospital-based surveillance to estimate the incidence of pertussis requiring hospitalization among infants in Germany and to determine the proportion of infants hospitalized with pertussis too young to have been vaccinated.

Methods: Enhanced surveillance was implemented within a nationwide hospital surveillance network (ESPED). We defined cases as children less than 1 year of age hospitalized due to laboratory-confirmed pertussis with disease onset from 01/07/2013-30/06/2015. We matched cases to those ascertained in the national statutory notification system, and estimated incidence using capture-recapture methodology.

Results: The estimated annual incidence of pertussis requiring hospitalization in infants was 52/100,000 infants ( $95 \%$ confidence interval [CI] $48-57 / 100,000$ ), with $39 \%$ under-reporting to the national notification system. During the two epidemiologic years under-reporting decreased from $46 \%$ to $32 \%$ and was lower in eastern than western federal states $(21 \% \mathrm{vs}$. $40 \%)$. Within ESPED, 154 of 240 infants (64\%) were younger than or still at the age recommended for the first vaccine dose; 55 (23\%) could have received one or more vaccine doses. Median length of hospitalization was 9 days (IQR 5-13 days) and 18\% required intensive care treatment.

Conclusions: Our study revealed a high burden of pertussis in infants with marked under-reporting, especially in western federal states where notification was only recently established. Strategies for the prevention of severe pertussis.
\end{abstract}

Key Words: pertussis, incidence, infants, hospitalization, capture-recapture

(Pediatr Infect Dis J 2018;37:119-125)

W hooping cough, also referred to as pertussis, is a highly contagious respiratory disease primarily caused by the bacterium Bordetella pertussis and more rarely by Bordetella parapertussis, which generally causes milder disease. ${ }^{1,2}$ Only the former is vaccine preventable. The disease is most severe in unvaccinated or incompletely vaccinated infants, who may develop apnea, seizures secondary to hypoxia, pulmonary hypertension, pneumonia or otitis media. ${ }^{2}$

Accepted for publication April 5, 2017.

From the *Immunization Unit, Department of Infectious Disease Epidemiology, Robert Koch Institute (RKI), Berlin, Germany; †Postgraduate Training for Applied Epidemiology (PAE, German Field Epidemiology Training Programme), Robert Koch Institute, Berlin, Germany; †European Programme for Intervention Epidemiology Training (EPIET), European Centre for Disease Prevention and Control (ECDC), Stockholm, Sweden; and §Institute of Social Pediatrics and Adolescent Medicine, Division of Epidemiology, Ludwig-Maximilians-University of Munich, Munich, Germany.

The authors have no conflicts of interest or funding to disclose.

Address for correspondence: Anika Schielke, PhD, Robert Koch Institute (RKI), Berlin, Germany. E-mail schielkea@rki.de.

Copyright (C) 2017 Wolters Kluwer Health, Inc. All rights reserved.

ISSN: 0891-3668/18/3702-0119

DOI: $10.1097 /$ INF.0000000000001698
Cyclical increases in pertussis incidence continue to be described every 3 to 5 years in most developed countries despite high vaccination coverage. This is explained largely by waning immunity both after vaccination and after natural infection..$^{3-6}$ There is evidence for more rapid waning of immunity after vaccination with acellular compared with whole cell pertussis vaccines. ${ }^{7,8}$ In Germany, acellular vaccines were introduced in 1995 and rapidly replaced whole cell vaccines. Infection of previously vaccinated persons may be asymptomatic or cause significantly milder illness ${ }^{9}$ and may, thus, go undiagnosed but may nonetheless result in transmission of pertussis to infants. ${ }^{9-11}$

The German Standing Committee on Vaccination (STIKO) recommends primary pertussis vaccination with vaccines containing high concentrations of pertussis antigens at age 2, 3 and 4 months followed by a booster at 11-14 months. Further boosters are recommended at school entry, in adolescence and in adults using vaccines containing lower concentrations of pertussis antigens. To further protect infants, especially those too young to be vaccinated, a cocoon strategy that recommends vaccination of women planning pregnancy, as well as household contacts and caregivers of infants, was adopted in 2004. ${ }^{12}$

Notification of pertussis was obligatory in the 5 eastern federal states of Germany according to a uniform case definition since 2002, but nationwide notification according to the Protection Against Infection Act was not implemented until March 29, 2013. ${ }^{13}$ Approximately half of all infants notified with pertussis are reported to have been hospitalized, ${ }^{14}$ which we consider an indicator of severe disease.

Knowledge of disease burden in infants, including the proportion of cases too young for vaccination, is essential for evaluation and possible adaptation of the current pertussis vaccination strategy. To estimate the true burden of severe pertussis in infants and to quantify the degree of underreporting in the national statutory reporting system in Germany, we conducted prospective hospital-based enhanced surveillance over 2 years and applied capturerecapture methodology. 15,16

\section{MATERIALS AND METHODS}

\section{Data Sources for Capture-Recapture Analysis National Statutory Notification System (SurvNet)}

Physicians and laboratories are required to report suspected and confirmed cases of pertussis to local health authorities (LHA). Only data from cases fulfilling the laboratory criteria of the national surveillance case definition ${ }^{17}$ are then electronically forwarded via the state health departments to the Robert Koch Institute.

\section{Nationwide Hospital Network for the Surveillance of Rare Pediatric Diseases}

All pediatric hospitals were invited to participate in this network from 1992 onwards. ${ }^{18}$ Each month, hospitals receive a reporting form asking for the number of cases hospitalized because of one of up to 12 different diseases according to the respective study case definition. From July 1, 2013 to June 30, 2015, this included 
pertussis in infants. Cases were defined as infants under 1 year of age hospitalized because of an acute $B$. pertussis infection confirmed by polymerase chain reaction, culture or serologic testing for pertussis toxin antibodies. For each reported case, the attending physician was asked to complete a standardized questionnaire and return it to the ESPED study office, which forwarded it to the Robert Koch Institute.

\section{Matching of Cases}

Data on month and year of birth, as well as sex of the child, district and federal state of residence, dates of disease onset and hospitalization, vaccination status, clinical findings and results of laboratory testing were available from both data sources, although more detail was available for the latter 2 from the active surveillance in the hospital network. If either the date of hospitalization or illness onset was missing in cases captured by ESPED, we estimated these as follows: we calculated the median interval between these 2 dates for all cases without missing data. We then estimated the date of hospitalization as the date of illness onset plus this median interval or the date of illness onset as the date of hospitalization minus this median interval. For cases captured by the national surveillance system with missing dates of disease onset or hospitalization, these were calculated analogously based on the median time between date of notification and either date of illness onset or date of hospitalization.

SurvNet and ESPED records were matched if the following criteria were fulfilled:

1. Month and year of birth AND

2. Sex

AND

3. District of residence (if missing, district of hospital) AND

4a. Date of hospitalization ( \pm 7 days; if missing, estimated date of hospitalization)

OR

4b. Disease onset ( \pm 14 days; if missing, estimated disease onset)

For the following analysis, we included cases from both data sources if they were younger than 1 year of age and hospitalized because of a laboratory-confirmed acute $B$. pertussis infection with disease onset from July 1, 2013 to June 30, 2015.

\section{Statistical Analysis}

We performed a descriptive analysis of the notified infants with pertussis according to age, sex, vaccination status, clinical manifestations and complications, length of hospital stay and treatment. As only month and year of birth were available to comply with data protection requirements, calculation of age in months was imprecise. We assumed the day of birth to be the 15 th of the month, meaning our age estimates could deviate by \pm 1 month.

We applied capture-recapture calculation (CRC) to estimate the total number of cases (Fig. 1) and their 95\% confidence intervals (CI) using Chapman's Nearly Unbiased Estimator. ${ }^{19,20}$ Incidences were calculated using official population data for Germany, 2014, from the Federal Statistical Office. We defined underreporting as the percentage difference between the CRC estimate and the incidence calculated based on the number of cases captured by the statutory or hospital-based surveillance systems, respectively.

We investigated whether validating the hospitalization status of cases reported to the national surveillance system influenced our

$$
n=\frac{(L 1+1) x(L 2+1)}{d+1}-1
$$

$\begin{array}{cl}\text { Abbreviation } & \text { Number of cases: } \\ \text { L1 } & \text { from data source 1 } \\ \text { L2 } & \text { from data source 2 } \\ \text { d } & \text { from both sources } \\ \text { m } & \text { not found in any data source } \\ \text { n } & \text { estimated as Nearly Unbiased Estimator [Chapman, 1951] }\end{array}$

FIGURE 1. Formula used for capture-recapture calculations.

CRC estimates as follows: we asked LHA staff to perform a retrospective validation of the hospitalization status in SurvNet for cases notified in 2013 and 2014 if the current hospitalization status was classified as "missing" or "not hospitalized". We then compared results of capture-recapture analysis performed using validated and unvalidated data. In addition, we stratified our CRC analysis by study year, region (eastern vs. western states) and age $(<3$ months vs. 3-11 months) to evaluate the consistency of estimates (variable catchability) by comparing crude estimates with the sum of the respective stratum estimates as recommended by Hook and Regal. ${ }^{21}$

\section{Ethics}

The study was approved by the local Ethics Committee of Charité Medical University, Berlin (EA2/080/13).

\section{RESULTS}

\section{Description of Cases and Comparison of the Two Data Sets SurvNet}

In the study period, 439 cases of pertussis in infants requiring hospitalization were reported to the national surveillance system, described in detail in Table 1. Briefly, the median age in months of the infants with plausible data $(n=437)$ was 3 months (interquartile range: $2-5$ months). The proportion of infants with a calculated age under 3 months, and, thus, younger than or still at the age recommended for the first vaccine dose, was $38 \%(n=165)$. The majority of the pertussis cases were laboratory confirmed by polymerase chain reaction. Median length of the reported hospital stay was 5 days. The most commonly reported symptom was cough, with paroxysmal attacks in about half and inspiratory stridor and apnea in about a quarter of the cases. No infant death was reported.

\section{Nationwide Hospital Network for the Surveillance of Rare Pediatric Diseases}

According to the Association of Pediatric Hospitals and Pediatric Hospital Departments, there were 360 (2013) and 356 (2014) pediatric hospitals or pediatric hospital departments in Germany (Personal communication, GKinD, 2016). Data for 2015 were not available at the time of writing. A total of 346 hospitals hospital departments participated in ESPED during the study period ( $>95 \%$ ). Response was received from $97 \%$ of participating hospitals/hospital departments for at least one of the months.

Over the study period, the participating hospitals reported a total of 247 pertussis cases to ESPED. Physicians completed the questionnaire for $240(97 \%)$ of these, which were, thus, included in the analysis and are described in Table 1. Briefly, median age was 2 months (interquartile range: 1-3 months), and the proportion of infants with a calculated age under 3 months, that is, 
TABLE 1. Characteristics of Infants Hospitalized Due to Pertussis Captured by the Data Sources SurvNet and ESPED and of the Cases for Which Matching Was Possible

\begin{tabular}{|c|c|c|c|c|c|c|c|c|c|}
\hline \multirow[b]{2}{*}{ Characteristics } & \multicolumn{3}{|c|}{ SurvNet } & \multicolumn{3}{|c|}{ ESPED } & \multicolumn{3}{|c|}{ Matched Cases } \\
\hline & $\mathrm{N}$ & $\%$ & Median & $\mathrm{N}$ & $\%$ & Median & $\mathrm{N}$ & $\%$ & Median \\
\hline Number of cases in total & 439 & & & 240 & & & 147 & & \\
\hline Age (months) & 437 & & $\begin{array}{l}3 \text { months } \\
\text { (IQR 2-5) }\end{array}$ & 239 & & $\begin{array}{l}2 \text { months } \\
\text { (IQR } 1-3)\end{array}$ & 147 & & $\begin{array}{l}2 \text { months } \\
\text { (IQR 1-3) }\end{array}$ \\
\hline Infants under 2 months of age & 85 & $19 \%$ & & 100 & $42 \%$ & & 65 & $44 \%$ & \\
\hline Infants 2 months of age & 80 & $18 \%$ & & 54 & $23 \%$ & & 31 & $21 \%$ & \\
\hline Infants probably too young for vaccination (under 3 months) & 165 & $38 \%$ & & 154 & $64 \%$ & & 96 & $65 \%$ & \\
\hline Proportion of girls & 218 & $50 \%$ & & 116 & $48 \%$ & & 75 & $51 \%$ & \\
\hline Reported from hospitals in western states & 385 & $88 \%$ & & 217 & $90 \%$ & & 129 & $88 \%$ & \\
\hline Duration of hospital stay (days) & 100 & & $\begin{array}{c}5 \text { days }(\mathrm{IQR} \\
3-9 ; \text { range } \\
1-29)\end{array}$ & 235 & & $\begin{array}{c}9 \text { days (IQR } \\
5-13 ; \text { range } \\
1-44)\end{array}$ & 144 & & $\begin{array}{c}9 \text { days (IQR } \\
5-13 ; \text { range } \\
1-44)\end{array}$ \\
\hline PCR & 335 & $76 \%$ & & 228 & $95 \%$ & & 143 & $97 \%$ & \\
\hline Serology & $42^{*}$ & $10 \%$ & & $10 \dagger$ & $4 \%$ & & 3 & $2 \%$ & \\
\hline Culture & 12 & $3 \%$ & & 2 & $1 \%$ & & 1 & $1 \%$ & \\
\hline No specification of laboratory method & 50 & $11 \%$ & & & & & & & \\
\hline Cough & 370 & $84 \%$ & & 238 & $99 \%$ & & 146 & $99 \%$ & \\
\hline With paroxysmal attacks & 185 & $50 \%$ & & 181 & $76 \%$ & & 110 & $75 \%$ & \\
\hline With inspiratory stridor & 77 & $21 \%$ & & 35 & $15 \%$ & & 21 & $14 \%$ & \\
\hline Apnea & 105 & $24 \%$ & & 79 & $33 \%$ & & 47 & $32 \%$ & \\
\hline Time (days) between first and last apnea episode & & & NA & 53 & & $\begin{array}{c}10 \text { days (IQR } \\
4-14 ; \text { range } \\
0-32)\end{array}$ & 32 & & $\begin{array}{c}10 \text { days }(\mathrm{IQR} \\
6-15 ; \text { range } \\
0-31)\end{array}$ \\
\hline Pneumonia & & NA & & 30 & $13 \%$ & & 16 & $11 \%$ & \\
\hline Intensive care & & NA & & 42 & $18 \%$ & & 23 & $16 \%$ & \\
\hline Deaths & 0 & & & 0 & & & 0 & & \\
\hline
\end{tabular}

*Twenty-two cases were confirmed with only a single elevated pertussis toxin antibody measurement; of these, 3 had received 1 dose of aP vaccine.

$\dagger$ Eight cases were confirmed with only a single elevated pertussis toxin antibody measurement; of these, 2 had received 1 dose of aP vaccine.

aP indicates pertussis antigen; ESPED, Nationwide Hospital Network for the Surveillance of Rare Pediatric Diseases; IQR, interquartile range; NA, data not available; PCR, polymerase chain reaction.

younger than or still at the age recommended for the first vaccine dose, was $64 \%(n=154)$ and, thus, higher than among the statutorily notified cases. Median length of the hospital stay was 9 days (interquartile range: $5-13$ days; range: $1-44$ days; $n=235$ ). The predominant symptom was cough in $99 \%(n=238)$. Of infants with cough, $76 \%(\mathrm{n}=181)$ had paroxysmal attacks typical for whooping cough and $15 \%(n=35)$ inspiratory stridor. Apnea was observed in one third of the infants $(n=79)$ with a median duration of 10 days. Pneumonia was reported in $13 \%(n=30)$, and $18 \%(n=42)$ required intensive care treatment. An additional $12 \%$ $(n=29)$ required cardiorespiratory monitoring. Additional symptoms were reported for $58 \%(n=140)$ of the infants. These were mainly other respiratory symptoms $(85 \%, n=120)$ such as dyspnea, tachypnea, cyanosis, low oxygen saturation and posttussive vomiting or general symptoms $(24 \%, n=33)$ such as somnolence, fever, loss of appetite and refusal to drink. No child developed otitis media, seizures, hypoxic encephalitis or died. Standard treatment was erythromycin $(68 \% ; n=162)$.

Of the 240 infants, $180(75 \%)$ had never been vaccinated against pertussis (Table 2). At least 55 infants with known age (47 unvaccinated and 8 vaccinated with fewer doses than recommended for age) could have received at least one (further) vaccine dose ( $23 \%$ of infants with known age; Table 2$)$.

\section{Matching of Cases}

We were able to match 147 ESPED cases to SurvNet cases. Based on our predefined matching criteria, 133 matches were entirely unambiguous. Case pairs that did not fulfill the matching criteria, but for which matching appeared probable $(n=18)$, were individually assessed, and 14 were judged to match. Characteristics of matched cases were very similar to those of all ESPED cases (Table 1).

\section{Estimated Incidences}

By CRC, we estimated a crude annual incidence of 50 pertussis cases per 100,000 infants (95\% CI: 46-54/100,000; Table 3 ).

The estimated nationwide incidence changed only slightly over time. In the first observed epidemiologic year (mid-2013 to mid2014), incidence was estimated at 52 cases $/ 100,000$ infants $(95 \% \mathrm{CI}$ : $46-59 / 100,000$ infants), decreasing to 49 (95\% CI: $44-54)$ in the second epidemiologic year (mid-2014 to mid-2015). In contrast, our statutory surveillance system indicated an increasing incidence during the same time period ( 28 to $33 / 100,000$ infants; Fig. 2). This was because of an increase in the number of notified cases in western (from 28 to 34 cases/100,000 infants) but not in eastern states, where incidence actually decreased from 28 to $24 / 100,000$.

We requested LHA to verify the hospitalization status for cases in 2013 and 2014 if hospitalization status was reported as "no" or "missing" ( $\mathrm{n}=341)$. LHA confirmed validation for 103 cases $(93$ originally reported as not hospitalized, 10 originally reported with missing hospitalization status). In 24 of the 103 cases, hospitalization status changed, in 19 cases to "hospitalized", thus fulfilling our case definition only after validation. Hospitalization status changed for a lower proportion of cases in $2014(18 \%$; 10/56) than in 2013 $(30 \%$; 14/47). However, our CRC estimates based on the data set with validated hospitalization status changed minimally compared with using the unvalidated hospitalization status $(49 / 100,000$ [validated] vs. 50/100,000 [unvalidated]).

\section{Estimated Underreporting}

Underreporting to the national notification system was $39 \%$ overall and was lower in eastern than in western federal states ( $21 \%$ vs. $40 \%$ ). It decreased from $46 \%$ in the first epidemiologic year to $32 \%$ in the second (Fig. 2). Despite greater underreporting in western federal states with improvement from the first to 
TABLE 2. Vaccination Status of the Cases Captured by ESPED According to the STIKO Recommendation

\begin{tabular}{|c|c|c|c|c|c|c|c|}
\hline $\begin{array}{l}\text { Age Group } \\
\text { (Months) }\end{array}$ & $\begin{array}{l}\text { STIKO Recommendation } \\
\text { for the Age Group }\end{array}$ & $\begin{array}{l}\text { Number of Infants } \\
\text { per Age Group }\end{array}$ & Unvaccinated & $\begin{array}{l}\text { Vaccinated } \\
1 \text { Dose }\end{array}$ & $\begin{array}{l}\text { Vaccinated } \\
2 \text { Doses }\end{array}$ & $\begin{array}{l}\text { Vaccinated } \\
3 \text { Doses }\end{array}$ & $\begin{array}{c}\text { Unknown Number of } \\
\text { Doses }\end{array}$ \\
\hline $0-1$ & No vaccination & 100 & $90 *$ & $0 *$ & $0 *$ & $0 *$ & $10 *$ \\
\hline 2 & 1 dose possible & 54 & $43 \dagger$ & $5 \dagger$ & 0 & 0 & $6 \ddagger$ \\
\hline 3 & At least 1 dose & 31 & $16 \S$ & $10 \dagger$ & $2 \dagger$ & 0 & $3 \ddagger$ \\
\hline 4 & At least 2 doses & 21 & $14 \S$ & $3 \S$ & $2 \dagger$ & $0 \dagger$ & $2 \ddagger$ \\
\hline 5 & Three doses & 13 & $10 \S$ & $1 \S$ & $0 \S$ & $0 \dagger$ & $2 \ddagger$ \\
\hline $6+$ & Three doses & 20 & $7 \S$ & $3 \S$ & $1 \S$ & $3 \dagger$ & $6 \ddagger$ \\
\hline Unknown age & Not possible & 1 & $0 \ddagger$ & $0 \div$ & $0 \ddagger$ & $0 \ddagger$ & $1 \ddagger$ \\
\hline Sum & & 240 & 180 & 22 & $\begin{array}{c}5 \\
30\end{array}$ & 3 & 30 \\
\hline
\end{tabular}

*Number of infants too young to be vaccinated $(\mathrm{n}=100)$.

$\dagger$ Number of infants vaccinated according to the STIKO recommendation for the age group $(\mathrm{n}=65)$.

$\$$ Number of infants for whom vaccination status could not be assessed $(\mathrm{n}=20)$.

$\S$ Number of infants NOT vaccinated according to the STIKO recommendation for the age group ( $\mathrm{n}=55)$.

ESPED indicates Nationwide Hospital Network for the Surveillance of Rare Pediatric Diseases; STIKO, German Standing Committee on Vaccination.

the second study year, in stratified analyses according to time and region, the sum of the $\mathrm{CRC}$ estimates in the strata was similar to the crude CRC estimate. However, when we stratified for age (under 3 months vs. 3-11 months of age), the sum of the CRC estimates in the strata was higher compared with the crude estimate (Table 3).

\section{DISCUSSION}

Using a 2-source capture-recapture approach, we estimated a high incidence of severe pertussis in infants in Germany, higher in western than in eastern federal states. This is in line with hospital discharge statistics showing higher pertussis incidence in infants in western compared with those in eastern German states. ${ }^{22}$ A higher pertussis disease burden would be in keeping with observed lower vaccination coverage in western states, most marked in adolescents. ${ }^{23-25}$ Our results thus indicate that the lower incidences in most western compared with most eastern states as based on national statutory surveillance data available since $2013^{14}$ are due to underreporting and possibly under-ascertainment related to the only recent implementation of statutory notification in western Germany. Indeed, the degree of underreporting was markedly higher in western than in eastern states, although this improved over the course of the study.

The incidence of pertussis in infants based on cases notified to the statutory surveillance system in eastern states (not corrected for underreporting) was 2- to 3-fold lower during our study period (annual incidence 2013-2015: 31 cases/100,000 infants) than during the last cyclic peak in 2011-2012, when the incidence in infants peaked at 95 cases/100,000 infants. ${ }^{22}$ Thus, taking this and the estimated underreporting into account, an incidence well over 100 hospitalizations/100,000 infants would be expected in epidemic years.

We hypothesize that underreporting and under-ascertainment in older age groups with typically milder disease may be even higher because these patients are less likely to seek medical care and, even when they do, may not be tested for pertussis.

The severity of pertussis in infants, reflected in prolonged hospitalization and requirement for intensive care in a high proportion of affected infants, together with the high incidence estimated in our study during an interepidemic period call for the implementation of further preventive measures. A high proportion of our cases was younger than or still at the age recommended for the first pertussis vaccine dose, that is, $<3$ months of age. In the United Kingdom, maternal vaccination introduced in response to an increase in infant deaths because of pertussis in 2012 was shown to have an effectiveness of $91 \%$ for preventing pertussis in infants under 3 months of age. ${ }^{26}$ In addition, a recent study from the United States found a relative effectiveness of $85 \%$ for maternal vaccination in pregnancy versus postpartum maternal vaccination. ${ }^{27} \mathrm{~A}$ number of countries have introduced maternal vaccination during pregnancy into their vaccination programs. ${ }^{26,28-32}$ While maternal antibodies of mothers who received vaccination antenatally may interfere with the immune response to routine vaccination of their infants, this effect appears to be small ${ }^{33,34}$ and was no longer significant after

TABLE 3. Incidence Estimates and Underreporting Based on CRC Stratified by Time, Region and Age Group

\begin{tabular}{|c|c|c|c|c|c|c|c|c|c|}
\hline & $\begin{array}{l}\text { Cases in } \\
\text { SurvNet }\end{array}$ & $\begin{array}{l}\text { Cases in } \\
\text { ESPED }\end{array}$ & $\begin{array}{c}\text { Matching } \\
\text { Cases }\end{array}$ & $\begin{array}{c}\text { CRC } \\
\text { Case } \\
\text { Estimate }\end{array}$ & Total & $\begin{array}{c}\text { Annual CRC } \\
\text { Case } \\
\text { Estimate }\end{array}$ & $\begin{array}{l}\text { Annual CRC Incidence } \\
\text { Estimate per 100,000 } \\
(95 \% \text { CI })\end{array}$ & $\begin{array}{l}\text { Underre- } \\
\text { porting to } \\
\text { SurvNet }\end{array}$ & $\begin{array}{l}\text { Underreporting } \\
\text { to ESPED }\end{array}$ \\
\hline Crude & 439 & 240 & 147 & 715 & & 358 & $50(46-54)$ & $39 \%$ & $66 \%$ \\
\hline \multicolumn{10}{|l|}{ Stratified by time } \\
\hline July 2013 to June 2014 & 203 & 126 & 68 & 374 & $722^{*}$ & 374 & $52(46-59)$ & $46 \%$ & $66 \%$ \\
\hline July 2014 to June 2015 & 236 & 114 & 77 & 348 & & 348 & $49(44-54)$ & $32 \%$ & $67 \%$ \\
\hline \multicolumn{10}{|l|}{ Stratified by region } \\
\hline Eastern states & 54 & 23 & 18 & 68 & $714 \dagger$ & 34 & $33(28-39)$ & $21 \%$ & $66 \%$ \\
\hline Western states & 385 & 217 & 129 & 646 & & 323 & $53(48-57)$ & $40 \%$ & $66 \%$ \\
\hline \multicolumn{10}{|l|}{ Stratified by age } \\
\hline $0-2$ months & 209 & 154 & 87 & 369 & $777 \ddagger$ & 184 & $103(92-114)$ & $43 \%$ & $58 \%$ \\
\hline 3-11 months & 227 & 85 & 47 & 408 & & 204 & $38(32-44)$ & $44 \%$ & $79 \%$ \\
\hline
\end{tabular}

Point estimates for the annual incidence based on the sum of the respective strata (column "Total"):

*50,

$\doteqdot 50$;

$\$ 54$ cases $/ 100,000$ inhabitants.

ESPED indicates Nationwide Hospital Network for the Surveillance of Rare Pediatric Diseases. 


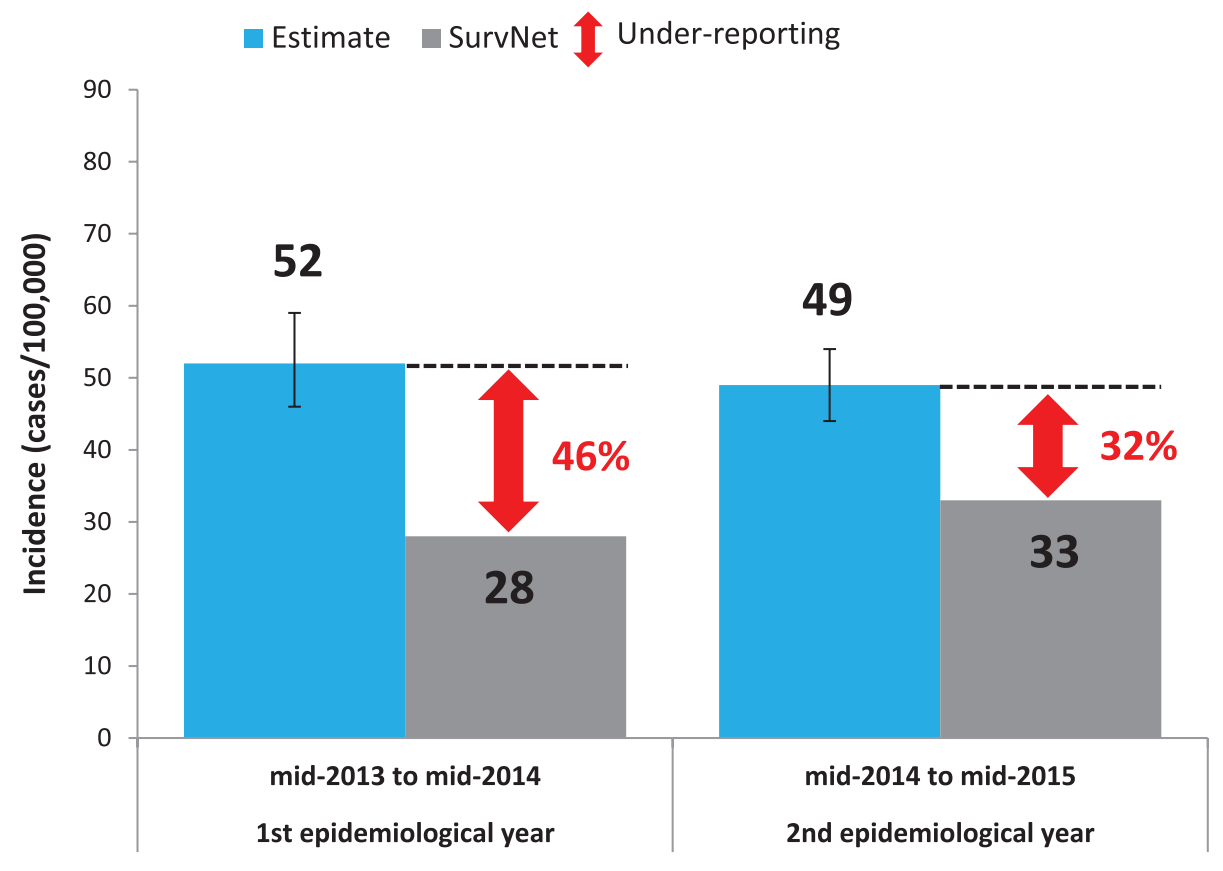

FIGURE 2. Comparison

of incidences of pertussis hospitalizations in infants estimated using capture-recapture methodology and based on SurvNet data in the 2 epidemiologic years 2013-2014 and 2014-2015 in Germany. Underreporting is highlighted by the red arrow. full color we may have underestimated incidence. The high degree of underreporting to ESPED suggests that this was not the case. Overestimation would result if reporting to one system made reporting to the other less likely (negative dependency). However, as not only clinicians, but also laboratories are required to notify cases to SurvNet, and laboratory notification is largely automated, this scenario also seems unlikely. Finally, the prerequisite of homogenous capture probabilities across all individuals in the population was not always met in our study. Although cases from the 2 parts of Germany were equally likely to be reported to ESPED, we observed a lower reporting probability to the statutory surveillance system from western states, where notification only recently became mandatory. We also found a higher degree of underreporting to ESPED for cases $\geq 3$ months of age than for younger cases. Nonetheless, in our analyses stratified by region and study year, the incidence estimates based on the sum of stratified estimates differed only marginally from the crude estimate (Table 3). Results of our stratified analysis by age, however, suggest that our crude estimate may be an underestimate, with a point estimate for annual pertussis incidence for infants based on the sum of the cases in the strata of 54 hospitalizations/100,000 (Table 3). Thus, overall, we believe the results of our CRC analysis provide a conservative estimate of pertussis requiring hospitalization in infancy in Germany. Our estimated crude incidence of 50 hospitalizations/100,000 infants was also higher than the incidence of hospitalizations for pertussis in infants calculated based on official aggregated hospital discharge statistics $^{53}$ - a mean of 300 pertussis hospitalizations annually from 2013 to 2015 or 42 hospitalizations/100,000 infants. However, the difference was less than that compared with incidence based on statutory surveillance data (Fig. 2). In any case, available aggregate hospital discharge data do not provide important information required for a comprehensive understanding of disease burden in infants, such as age in months, diagnostic confirmation, vaccination status or disease severity.

One final limitation of our study involves infants diagnosed with only a single measurement of elevated PT antibodies. ${ }^{54,55}$ However, because polymerase chain reaction or cultural confirmation of pertussis are preferred in infants, this applies only for a small proportion of infants. High levels of maternal antibodies are only 
rarely seen in infants whose mothers did not receive a pertussis antigen vaccine during pregnancy, ${ }^{56,57}$ and only 3 of the serologically confirmed cases had been vaccinated (Table 1 ).

In conclusion, we found a high incidence of pertussis requiring hospitalization in infants during an interepidemic period of the pertussis cycle. A high proportion of cases was too young to have been protected through pertussis vaccination recommended from 2 months of age. In view of the poor implementation of the cocoon strategy in Germany and the observed effectiveness of maternal vaccination in other countries, these findings suggest that maternal vaccination would be an effective strategy to prevent pertussis in young infants in Germany and should, therefore, be assessed by STIKO. In addition, about a quarter of the affected infants were old enough to have received at least their first pertussis vaccine dose, highlighting the importance of timely vaccination in infancy. Finally, this study should be repeated in a few years to confirm the anticipated further improvement in case ascertainment in western federal states.

\section{REFERENCES}

1. Kretzschmar M, Teunis PF, Pebody RG. Incidence and reproduction numbers of pertussis: estimates from serological and social contact data in five European countries. PLoS Med. 2010;7:e1000291.

2. Mattoo S, Cherry JD. Molecular pathogenesis, epidemiology, and clinical manifestations of respiratory infections due to Bordetella pertussis and other Bordetella subspecies. Clin Microbiol Rev. 2005;18:326-382.

3. Klein NP, Bartlett J, Fireman B, et al. Waning Tdap effectiveness in adolescents. Pediatrics. 2016;137:e20153326.

4. Koepke R, Eickhoff JC, Ayele RA, et al. Estimating the effectiveness of tetanus-diphtheria-acellular pertussis vaccine (Tdap) for preventing pertussis: evidence of rapidly waning immunity and difference in effectiveness by Tdap brand. J Infect Dis. 2014;210:942-953.

5. Wendelboe AM, Van Rie A, Salmaso S, et al. Duration of immunity against pertussis after natural infection or vaccination. Pediatr Infect Dis J. 2005;24(5 supp1):S58-S61.

6. Tartof SY, Lewis M, Kenyon C, et al. Waning immunity to pertussis following 5 doses of DTaP. Pediatrics. 2013;131:e1047-e1052.

7. Sheridan SL, Ware RS, Grimwood K, et al. Reduced risk of pertussis in whole-cell compared to acellular vaccine recipients is not confounded by age or receipt of booster-doses. Vaccine. 2015;33:5027-5030.

8. Liko J, Robison SG, Cieslak PR. Priming with whole-cell versus acellular pertussis vaccine. $N$ Engl J Med. 2013;368:581-582.

9. Barlow RS, Reynolds LE, Cieslak PR, et al. Vaccinated children and adolescents with pertussis infections experience reduced illness severity and duration, Oregon, 2010-2012. Clin Infect Dis. 2014;58:1523-1529.

10. Trainor EA, Nicholson TL, Merkel TJ. Bordetella pertussis transmission. Pathog Dis. 2015;73(8):ftv068.

11. Wiley KE, Zuo Y, Macartney KK, et al. Sources of pertussis infection in young infants: a review of key evidence informing targeting of the cocoon strategy. Vaccine. 2013;31:618-625.

12. STIKO. Mitteilung der Ständigen Impfkommission am Robert Koch-Institut (RKI). Empfehlungen der Ständigen Impfkommission (STIKO) am Robert Koch-Institut - 2016/2017. Epid Bull. 2016;34(2016):301-338.

13. Robert Koch Institut. Falldefinitionen zur Übermittlung von Erkrankungsund Todesfällen sowie von Erregernachweisen von Mumps, Pertussis, Röteln und Varizellen. Bundesgesundheitsblatt - Gesundheitsforschung Gesundheitsschutz, 2013;56(7):1003-1016.

14. Robert Koch Institut, Infektionsepidemiologisches Jahrbuch meldepflichtiger Krankheiten für 2014. Vol. http://www.rki.de/DE/Content/Infekt/Jahrbuch/ Jahrbuecher/2014.html?nn=2374622. 2015, Berlin: Mercedes-Druck.

15. International Working Group for Disease Monitoring and Forecasting, Capture-recapture and multiple-record systems estimation I: history and theoretical development. Am J Epidemiology, 1995;142(10):1047-1058.

16. International Working Group for Disease Monitoring and Forecasting, Capture-recapture and multiple-record systems estimation II: Apllications in human diseases. Am J Epidemiol, 1995;142(10):1059-1068.

17. Robert Koch-Institut Falldefinitionen des Robert Koch-Instituts zur Übermittlung von Erkrankungs- oder Todesfällen und Nachweisen von Krankheitserregern 2015. Wittenberg, Germany: Elbe Druckerei Wittenberg $\mathrm{GmbH}$.
18. von Kries R, Heinrich B, Hermann M, Pädiatrische Epidemiologie in Deutschland: Forschungsinstrument ESPED Erhebungseinheit für seltene pädiatrische Erkrankungen in Deutschland. Monatsschrift fur Kinderheilkunde. 2001;149:1191-1197.

19. Chapman DG. In: Loève M, Kuznets GM, Lehmann EL, Neyman J, eds. Some Properties of the Hypergeometric Distribution With Applications to the Zoological Sample Censuses. Berkely and Los Angeles, CA: University of California Statistical Publications; 1951:131-160.

20. Mellou K, Sideroglou T, Kallimani A, et al. Evaluation of underreporting of salmonellosis and shigellosis hospitalised cases in Greece, 2011: results of a capture-recapture study and a hospital registry review. BMC Public Health. 2013;13:875.

21. Hook EB, Regal RR. Effect of variation in probability of ascertainment by sources ("variable catchability") upon "capture-recapture" estimates of prevalence. Am J Epidemiol. 1993;137:1148-1166.

22. Robert-Koch-Institut, Zur Situation bei ausgewählten Infektionskrankheiten in Deutschland Keuchhusten-Erkrankungen in den neuen Bundesländern, 2002 bis 2012. Epidemiologisches Bulletin, 2014;1:1-11.

23. Poethko-Müller C, Schmitz R. [Vaccination coverage in German adults: results of the German Health Interview and Examination Survey for Adults (DEGS1)]. Bundesgesundheitsblatt Gesundheitsforschung Gesundheitsschutz. 2013;56:845-857.

24. Poethko-Müller C, Kuhnert R, Schlaud M. [Vaccination coverage and predictors for vaccination level. Results of the German Health Interview and Examination Survey for Children and Adolescents (KiGGS)] Bundesgesundheitsblatt Gesundheitsforschung Gesundheitsschutz. 2007;50:851-862

25. Ellsäßer G, Trost-Brinkhues G. [Vaccination among students in grades 6-10, 2011 - a comparison of German states: need for action for a targeted nationwide immunisation strategy]. Gesundheitswesen. 2013;75:705-713.

26. Amirthalingam G, Andrews N, Campbell H, et al. Effectiveness of maternal pertussis vaccination in England: an observational study. Lancet. 2014;384:1521-1528.

27. Winter K, Nickell S, Powell M, et al. Effectiveness of prenatal versus postpartum Tdap vaccination in preventing infant pertussis. Clin Infect Dis, 2016;64:3-8.

28. Centers for Disease Control and Prevention (CDC). Updated recommendations for use of tetanus toxoid, reduced diphtheria toxoid, and acellular pertussis vaccine (Tdap) in pregnant women--Advisory Committee on Immunization Practices (ACIP), 2012. MMWR Morb Mortal Wkly Rep, 2013;62(7):131-135.

29. Walls T, Graham P, Petousis-Harris H, et al. Infant outcomes after exposure to Tdap vaccine in pregnancy: an observational study. BMJ Open 2016;6:e009536.

30. World Health Organization, Pertussis vaccines: WHO position paper, August 2015--Recommendations. Vaccine. 2016;34(12):1423-1425.

31. Australian Technical Advisory Group on Immunisation (ATAGI), The Australian Immunisation Handbook 10th Edition (2015 update). Canberra: Australian Government Department of Health; 2015.

32. European Centre for Disease Prevention and Control, Shortage of Acellular Pertussis-Containing Vaccines and Impact on Immunisation Programmes in the EU/EEA-8 October 2015. Stockholm: European Centre for Disease Prevention and Control; 2015.

33. Hardy-Fairbanks AJ, Pan SJ, Decker MD, et al. Immune responses in infants whose mothers received Tdap vaccine during pregnancy. Pediatr Infect Dis J. 2013;32:1257-1260

34. Ladhani SN, Andrews NJ, Southern J, et al. Antibody responses after primary immunization in infants born to women receiving a pertussis-containing vaccine during pregnancy: single arm observational study with a historical comparator. Clin Infect Dis. 2015;61:1637-1644.

35. Maertens K, Hoang TT, Nguyen TD, et al. The effect of maternal pertussis immunization on infant vaccine responses to a booster pertussis-containing vaccine in Vietnam. Clin Infect Dis. 2016;63(suppl 4):S197-S204.

36. Munoz FM, Bond NH, Maccato M, et al. Safety and immunogenicity of tetanus diphtheria and acellular pertussis (Tdap) immunization during pregnancy in mothers and infants: a randomized clinical trial. JAMA. 2014;311:1760-1769.

37. Cherry JD. Editorial commentary: the effect of Tdap vaccination of pregnant women on the subsequent antibody responses of their infants. Clin Infect Dis. 2015;61:1645-1647.

38. Feunou PF, Mielcarek N, Locht C. Reciprocal interference of maternal and infant immunization in protection against pertussis. Vaccine. 2016;34:10621069 . 
39. Juretzko P, von Kries R, Hermann M, et al. Effectiveness of acellular pertussis vaccine assessed by hospital-based active surveillance in Germany. Clin Infect Dis. 2002;35:162-167.

40. Nilsson L, Lepp T, von Segebaden K, et al. Pertussis vaccination in infancy lowers the incidence of pertussis disease and the rate of hospitalisation after one and two doses: analyses of 10 years of pertussis surveillance. Vaccine. 2012;30:3239-3247.

41. Quinn HE, Snelling TL, Habig A, et al. Parental Tdap boosters and infant pertussis: a case-control study. Pediatrics. 2014;134:713-720.

42. Pesco P, Bergero P, Fabricius G, et al. Modelling the effect of changes in vaccine effectiveness and transmission contact rates on pertussis epidemiology. Epidemics. 2014;7:13-21.

43. de Greeff SC, Mooi FR, Westerhof A, et al. Pertussis disease burden in the household: how to protect young infants. Clin Infect Dis. 2010;50:1339-1345.

44. Kowalzik F, Barbosa AP, Fernandes VR, et al. Prospective multinational study of pertussis infection in hospitalized infants and their household contacts. Pediatr Infect Dis J. 2007;26:238-242.

45. Skoff TH, Kenyon C, Cocoros N, et al. Sources of infant pertussis infection in the United States. Pediatrics. 2015;136:635-641.

46. Böhmer MM, Hellenbrand W, Matysiak-Klose D, et al. [Pertussis vaccination coverage among adults in Germany]. Dtsch Med Wochenschr. 2013;138:1451-1457.

47. Bödeker B, Remschmidt C, Müters S, et al. [Influenza, tetanus, and pertussis vaccination coverage among adults in Germany]. Bundesgesundheitsblatt Gesundheitsforschung Gesundheitsschutz. 2015;58:174-181.

48. Lim GH, Deeks SL, Crowcroft N.S. A cocoon immunisation strategy against pertussis for infants: does it make sense for Ontario? Eurosurveillance. 2014;19(5):pii=20688.
49. Skowronski DM, Janjua NZ, Tsafack EP, et al. The number needed to vaccinate to prevent infant pertussis hospitalization and death through parent cocoon immunization. Clin Infect Dis. 2012;54:318-327.

50. Healy CM, Rench MA, Wootton SH, et al. Evaluation of the impact of a pertussis cocooning program on infant pertussis infection. Pediatr Infect Dis J. 2015;34:22-26.

51. Althouse BM, Scarpino SV. Asymptomatic transmission and the resurgence of Bordetella pertussis. BMC Med. 2015;13:146.

52. Hook EB, Regal RR. Capture-recapture estimation. Epidemiology. 1995;6:569-570.

53. Statistisches Bundesamt Krankenhausdiagnosestatistik-Pertussis http://www.gbe-bund.de/gbe 10/abrechnung.prc-abr-test$\log$ on $? \mathrm{p}-\mathrm{uid}=$ gast $\& \mathrm{p}-\mathrm{aid}=\& \mathrm{p}-\mathrm{sprache}=\mathrm{D} \& \mathrm{p}-$ knoten $=\mathrm{VR} \& \mathrm{p}-$ suchstring=pertussis, 2016.

54. European Centre for Disease Prevention and Control, Guidance and Protocol for the Use of Realtime PCR in Laboratory Diagnosis of Human Infection With Bordetella pertussis or Bordetella parapertussis. Stockholm: European Centre for Disease Prevention and Control; 2012.

55. Guiso N, Berbers G, Fry NK, et al.; EU Pertstrain group. What to do and what not to do in serological diagnosis of pertussis: recommendations from EU reference laboratories. Eur J Clin Microbiol Infect Dis. 2011;30:307312 .

56. Healy CM, Rench MA, Baker CJ. Importance of timing of maternal combined tetanus, diphtheria, and acellular pertussis (Tdap) immunization and protection of young infants. Clin Infect Dis. 2013;56:539-544.

57. Smallenburg LC, van Welie NA, Elvers LH, et al. Decline of IgG pertussis toxin measured in umbilical cord blood, and neonatal and early infant serum. Eur J Clin Microbiol Infect Dis. 2014;33:1541-1545. 